\title{
The Effects of Minimal Length, Maximal Momentum, and Minimal Momentum in Entropic Force
}

\author{
Zhong-Wen Feng, ${ }^{1}$ Shu-Zheng Yang, ${ }^{2}$ Hui-Ling Li, ${ }^{1,3}$ and Xiao-Tao $\mathrm{Zu}^{1}$ \\ ${ }^{1}$ School of Physical Electronics, University of Electronic Science and Technology of China, Chengdu 610054, China \\ ${ }^{2}$ Department of Astronomy, China West Normal University, Nanchong 637009, China \\ ${ }^{3}$ College of Physics Science and Technology, Shenyang Normal University, Shenyang 110034, China
}

Correspondence should be addressed to Zhong-Wen Feng; zwfengphy@163.com

Received 24 April 2016; Revised 25 June 2016; Accepted 11 July 2016

Academic Editor: Giulia Gubitosi

Copyright (C) 2016 Zhong-Wen Feng et al. This is an open access article distributed under the Creative Commons Attribution License, which permits unrestricted use, distribution, and reproduction in any medium, provided the original work is properly cited. The publication of this article was funded by SCOAP ${ }^{3}$.

\begin{abstract}
The modified entropic force law is studied by using a new kind of generalized uncertainty principle which contains a minimal length, a minimal momentum, and a maximal momentum. Firstly, the quantum corrections to the thermodynamics of a black hole are investigated. Then, according to Verlinde's theory, the generalized uncertainty principle (GUP) corrected entropic force is obtained. The result shows that the GUP corrected entropic force is related not only to the properties of the black holes but also to the Planck length and the dimensionless constants $\alpha_{0}$ and $\beta_{0}$. Moreover, based on the GUP corrected entropic force, we also derive the modified Einstein's field equation (EFE) and the modified Friedmann equation.
\end{abstract}

\section{Introduction}

The existence of thermodynamics of black holes is a great discovery for the foundations of physics [1-11]. This idea was proposed by Bekenstein who proved that the entropy of a black hole is $S=A k_{B} c^{3} / 4 \hbar G$, where $A$ is the horizon area, $k_{B}$ is the Boltzmann constant, $\hbar$ is the Planck constant, and $G$ is Newton's gravitational constant, respectively [2]. Later, based on the entropy of black hole, Hawking showed that the Schwarzschild (SC) black hole emits thermal radiation and the temperature of SC black hole is proportional to the surface gravity on the event horizon $\kappa$; namely, $T=\kappa / 2 \pi[5,6]$. Furthermore, in $[9,10]$, the authors proved that the entropy and the temperature of black hole satisfy the laws of thermodynamics. These discoveries indicate that the thermodynamics of black hole have profound connection with the gravity.

In order to investigate the deeper-seated relation between thermodynamics and the gravity, Jacobson assumed the spacetime as a kind of gas, its entropy is proportional to the area, then using the fundamental Clausius relation and the equivalence principle, he demonstrated that the Einstein field equation is nothing but a state equation of this kind of gas. Following this agreement, the Einstein field equation can be derived from the first law of thermodynamics together with the relation between the entropy and the horizon area of a black hole [12]. Subsequently, Padmanabhan pointed out that, in the static spherically symmetric spacetimes, the gravitational field equations on the horizon can be rewritten as a form of the ordinary first law of thermodynamics [13]. Inspired by Padmanabhan's idea, people found that Einstein's equation is a thermodynamic identity. Those works explain why the field equations should encode information of horizon thermodynamics [14-19].

In 2011, Verlinde proposed a remarkable new perspective on the relation between the gravity and the thermodynamics. Based on Sakharov's idea [20] and the holographic principle, Verlinde pointed out that the gravity is no longer a fundamental force; instead, it can be explained as an entropic force which arises from the change of information when material bodies move away from the screens of holographic systems [21]. In his paper, Verlinde showed various interesting results. For example, the the second law of Newton can be obtained by incorporating the entropic force with the Unruh temperature. Using the entropic force together with the holographic principle and the equipartition law of energy, one can yield Newton's law of gravitation. Moreover, 
an astounding discovery should be mentioned that Einstein's equation can be derived from the theory of entropic force. This new proposal of the gravity has received wide attention causing people to do many relevant works to discuss the entropic force [22-25].

On the other hand, a lot of works showed that the original thermodynamics of black holes would not be held when considering the quantum gravity effects [26-31]. Various theories of quantum gravity suggest the existence of a minimal observable length, which can be identified with the Planck scale. This view is supported by many Gedanken experiments and is applied to different physical systems [32-35]. The generalized uncertainty principle (GUP) is one of the most important theories, which is modified by the minimum measurable length. The applications of GUP has been widely studied [36-45]. In particular, the GUP effect on the micro black holes has been deeply discussed in [4648]. In [49], combining the GUP with the thermodynamics of black holes, the authors investigated the modified Hawking temperature and the entropy; their results showed that the GUP corrected thermodynamics of black holes are different from those of the original cases. The GUP can stop the Hawking radiation in the final stages of black holes' evolution and lead to the remnants of black holes. Therefore, the GUP is considered as a good tool to solve the information paradox problem of black holes. In [50-57], people have investigated the GUP corrected entropic force by using the GUP corrected thermodynamics. The implications of modified entropic force have been investigated in many contexts such as cosmology [50-53], Hamiltonians of the quantum systems in quasispace [54], quantum walk [55], and quarkonium binding [56, 57]. In fact, the expression of GUP is not unique. However, in most of the papers, the expression of GUP is limited to two forms. One form only has a minimal length $\Delta x \Delta p \geq \hbar\left[1+\alpha_{0}^{2} \ell_{p}^{2}(\Delta p)^{2}\right]$ [30]. The other form has a minimal length and a maximal momentum $\Delta x \Delta p \geq$ $\hbar\left[1-\alpha_{0} \ell_{p} \Delta p+\alpha_{0}^{2} \ell_{p}^{2}(\Delta p)^{2}\right]$ [58]. According to the previous works, it is interesting to raise the question whether it is possible to derive a general form of GUP which contains a minimal length, a minimal momentum, and a maximal momentum. Actually, in [59], the authors pointed out that the existence of a minimal length $\Delta x_{\min }$ comes from the fact that a string cannot probe its distances smaller than the length, and the maximal momentum $\Delta p_{\max }$ originated in the doubly special relativity (DSR) that predicts there exists an upper bound for the momentum of a particle. For the minimal momentum $\Delta p_{\min }$, it is well known that the notion of a plane wave does not appear in the a general curved spacetime; hence, it indicates that there exists a limit to the precision with which the corresponding momentum can be described. According to this phenomenon, one can express a nonzero minimal uncertainty in momentum measurement, that is, $\Delta p_{\text {min }}$. Taking these facts into account, Nozari and Saghafi introduced the most general form of GUP, which admits a minimal length, a minimal momentum, and a maximal momentum. The GUP is given by

$$
\Delta x \Delta p \geq \hbar\left[1-\alpha_{0} \ell_{p} \Delta p+\alpha_{0}^{2} \ell_{p}^{2}(\Delta p)^{2}+\beta_{0}^{2} \ell_{p}^{2}(\Delta x)^{2}\right]
$$

where $\Delta x$ and $\Delta p$ are the uncertainties for position and momentum, $\alpha_{0}$ and $\beta_{0}$ are dimensionless constants of the order of unity that depends on the details of the quantum gravity hypothesis, and $\ell_{p}=\sqrt{\hbar G / c^{3}} \approx 10^{-35} \mathrm{~m}$ is the Planck length, respectively [60]. From (1), one can easily obtain the minimal length $\Delta x_{\min }=\alpha_{0} \ell_{p}$, the minimal momentum $\Delta p_{\min }=2 \beta_{0} \ell_{p}$, and the maximal momentum $\Delta p_{\max }=[1+$ $\left.\sqrt{1-\left(1+\alpha_{0}^{2} \beta_{0}^{2} \ell_{p}^{4}\right)}\right] / \alpha_{0} \ell_{p}$. Under the standard limit, that is, $\Delta x \gg \ell_{p}$, (1) becomes the Heisenberg uncertainty principle (HUP). It is well known that GUP has a great effect on the thermodynamics of black holes and the entropic force that would bring many new results. Therefore, in this paper, using (1), we first investigate the modified thermodynamics of a black hole. Then, following Verlinde's viewpoint, the modified number of bits $N$ is obtained. The modified number of bits $N$ leads to the GUP corrected entropic force. Considering the GUP corrected entropic force, the gravitational force, the gravitational potential, Einstein's field equation, and the Friedmann equation are modified.

This paper is organized as follows. The quantum corrections to the entropy and Hawking temperature are derived in Section 2. According to Verlinde's theory, the GUP corrected entropic force is calculated in Section 3. In Sections 4 and 5, using the GUP corrected entropic force, the modified Einstein's field equation and the modified Friedmann equation is investigated. The last section is devoted to the conclusions.

\section{The GUP Impact on the Thermodynamics of a Black Hole}

In order to calculate the GUP impact on the thermodynamics of a black hole, one needs to solve (1) as a quadratic equation in $\Delta p$. The result is given by

$$
\begin{aligned}
\Delta p & \geq \frac{\Delta x+\hbar \alpha_{0} \ell_{p}}{2 \hbar \alpha_{0}^{2} \ell_{p}^{2}}\{1 \\
& \left.-\sqrt{1-\frac{4 \hbar^{2} \alpha_{0}^{2} \ell_{p}^{2}\left[1+\beta_{0}^{2}(\Delta x)^{2} \ell_{p}^{2}\right]}{\left(\Delta x+\hbar \alpha_{0} \ell_{p}\right)^{2}}}\right\},
\end{aligned}
$$

where we choose the negative-signed solution since only it can recover the HUP in the classical limit $\ell_{p} \rightarrow 0$. Employing the Taylor expansion, (2) can be rewritten as

$$
\begin{aligned}
\Delta p & \geq \frac{1}{\Delta x+\alpha_{0} \ell_{p}}\{1 \\
& \left.+\left[\beta_{0}^{2}(\Delta x)^{2}+\frac{\alpha_{0}^{2}}{\left(\Delta x+\alpha_{0} \ell_{p}\right)^{2}}\right] \ell_{p}^{2}+\mathcal{O}\left(\ell_{p}^{4}\right)\right\},
\end{aligned}
$$

where we are setting $\hbar=1$. As $[49,61]$ have pointed out, the uncertainty momentum $\Delta p$ can be defined as the energy $\omega$ of 
emitted photon from black hole. Therefore, based on (3), the low bound for the energy is

$$
\begin{aligned}
\omega & \geq \frac{1}{\Delta x+\alpha_{0} \ell_{p}}\left\{1+\left[\beta_{0}^{2}(\Delta x)^{2}+\frac{\alpha_{0}^{2}}{\left(\Delta x+\alpha_{0} \ell_{p}\right)^{2}}\right] \ell_{p}^{2}\right. \\
& \left.+\mathcal{O}\left(\ell_{p}^{4}\right)\right\} .
\end{aligned}
$$

Next, assuming an emitted particle with energy $\omega$ and size $R$, for any black hole absorbing or emitting this particle, the minimal change in the horizon area of a black hole can be expressed as

$$
\Delta A_{\min } \geq 8 \pi \omega R \ell_{p}^{2}
$$

In the classical model, people can set $R=0$. However, according to the arguments of [2], the size of a quantum particle cannot be smaller than $\Delta x$. Therefore, it implies the existence of a finite boundary given by

$$
\Delta A_{\min } \geq 8 \pi \omega \Delta x \ell_{p}^{2}
$$

By substituting (4) into inequality (6), then, using the relation of minimal length $\Delta x_{\min }=\alpha_{0} \ell_{p}$, the boundary turns out to be

$$
\begin{aligned}
& \Delta A_{\min } \\
& \quad \geq 4 \pi \ell_{p}^{2}\left\{1+\left[\beta_{0}^{2}(\Delta x)^{2}+\frac{\alpha_{0}^{2}}{(2 \Delta x)^{2}}\right] \ell_{p}^{2}+\mathcal{O}\left(\ell_{p}^{4}\right)\right\} .
\end{aligned}
$$

Now, consider the case that a photon is emitted by the SC black hole $[49,61,62]$. Near the event horizon of SC black hole, the position uncertainty of a photon is the order of the radius of the black hole; that is, $\Delta x=2 r_{s}$, where $r_{s}$ is the radius of SC black hole. According to the area of the SC black hole $A=4 \pi r_{s}^{2}$, the relation between $\Delta x$ and $A$ can be expressed as $(\Delta x)^{2}=4 r_{s}^{2}=A / \pi$. Substituting this relation into (7), the abovementioned equation can be rewritten as the following expression:

$$
\Delta A_{\text {min }} \simeq \lambda \ell_{p}^{2}\left[1+\left(\beta_{0}^{2} \frac{A}{\pi}+\frac{\pi \alpha_{0}^{2}}{4 A}\right) \ell_{p}^{2}+\mathcal{O}\left(\ell_{p}^{4}\right)\right],
$$

with $\lambda$ being an undetermined coefficient that is greater than $4 \pi$. In the previous works, people proved that the entropy of black holes depended on the area of horizon. Moreover, the ideas of information theory also showed the minimal increase of entropy is conjectured related to the value of the area $A$. According to $[2,63]$, the fundamental unit of entropy as one bit of information can be denoted as $\Delta S_{\min }=b=\ln 2$, so that one can easily obtain

$$
\begin{aligned}
\frac{d S}{d A} & =\frac{\Delta S_{\min }}{\Delta A_{\min }} \\
& =\frac{b}{\lambda \ell_{p}^{2}}\left[1+\left(\beta_{0}^{2} \frac{A}{\pi}+\frac{\pi \alpha_{0}^{2}}{4 A}\right) \ell_{p}^{2}+\mathcal{O}\left(\ell_{p}^{4}\right)\right]^{-1} .
\end{aligned}
$$

In accordance with the idea of entropy-area law, we obtain a constant $b / \lambda=k_{B} / 4$. Putting this constant into the abovementioned equation and expanding it and then integrating the result, the GUP corrected entropy is obtained as follows:

$$
\begin{aligned}
S= & \frac{A k_{B}}{4 \ell_{p}^{2}}\left\{1-\left[\frac{\alpha_{0}^{2} \pi}{4 A} \ln \left(\frac{A}{4 \ell_{p}^{2}}\right)+\frac{A \beta_{0}^{2}}{2 \pi}\right] \ell_{p}^{2}\right. \\
& \left.+\mathcal{O}\left(\ell_{p}^{4}\right)\right\} .
\end{aligned}
$$

It is clear that the GUP corrected entropy is proportional to the area of horizon $A$, Planck length $\ell_{p}$, and the dimensionless constants $\alpha_{0}$ and $\beta_{0}$. When ignoring $\alpha_{0}$ and $\beta_{0}$, (10) reduces to the original entropy of the black hole. Moreover, it can be found that the first correction term of (10) is logarithmic in $A, \ell_{p}$, and $\alpha_{0}$, which is coincident with previous findings [64$66]$. It should be noted that the second correction term is proportional to $\beta_{0}$ and goes like $A^{2}$; if $\beta_{0} \geq \sqrt{2 \pi / A}$, the second correction term is in principle larger than the leading term proportional to $A$. In order to avoid this paradoxical situation, it requires that $\beta_{0}<\sqrt{2 \pi / A}$. Meanwhile, one can calculate the GUP corrected Hawking temperature based on (10):

$$
\begin{aligned}
T & =\frac{\kappa}{8 \pi} \frac{d A}{d S} \\
& =\frac{\kappa}{2 \pi k_{B}} \ell_{p}^{2}\left[1+\left(\frac{A \beta_{0}^{2}}{\pi}+\frac{\pi \alpha_{0}^{2}}{4 A}\right) \ell_{p}^{2}+\mathcal{O}\left(\ell_{p}^{4}\right)\right],
\end{aligned}
$$

where $\kappa$ is the surface gravity of black holes. For the SC black hole, one sets $\kappa=1 / 4 M$, if one sets $\alpha_{0}=\beta_{0}=0$, the GUP corrected temperature reduces to the original Hawking temperature.

\section{The Modified Newton's Law of Gravitation due to the GUP}

In this section, we will investigate the GUP impact on Newton's law of gravitation. For revealing the entropic force, Verlinde used the holographic principle and the first law of thermodynamics. When a test particle approaches a holographic screen, the entropic force of a gravitational system is expressed as

$$
F \Delta x=T \Delta S
$$

where $F$ is the entropic force, $T$ and $\Delta S$ are the temperature and the change of entropy on holographic screen, and $\Delta x$ is the displacement of the particle from the holographic screen, respectively [21]. Equation (12) implies a nonzero force is proportional to a nonzero acceleration. Using the argument of Bekenstein, that is, the change of entropy associated with the information on the boundary $\Delta S=2 \pi k_{B}$, it is found that the change in the entropy near the holographic screen is linear in $\Delta x$ :

$$
\Delta S=\frac{2 \pi k_{B} m c \Delta x}{\hbar}
$$


where $\Delta x=\hbar / m c$ and $m$ and $\hbar$ are the mass of elementary component and the Planck constant, respectively. Equation (13) is reminiscent of the osmosis across a semipermeable membrane. Meanwhile, it should be noted that $\Delta S$ is proportional to the mass of the elementary component. In order to understand this idea, one can postulate that a particle near the holographic screen is made up of two or more subparticles and each subparticle leads to the associated change in entropy after displacement. Because the mass of the elementary component and the entropy are additive, it leads to the fact that $\Delta S$ is proportional to $m$. Based on the conclusions in [67], the horizon of black holes can be taken as a storage device for information. If one denotes the amount of information by $N$ bits, the information is proportional to the area $N=A c^{3} / G \hbar$. With the help of the entropy-area law $S=$ $A k_{B} c^{3} / 4 \hbar G$, the number of bits obeys the following relation:

$$
N=\frac{4 S}{k_{B}} .
$$

Obviously, the number of bits is proportional to the entropy. Substituting (10) into (14), the number of bits is modified as follows:

$$
\begin{aligned}
N & =\frac{A}{\ell_{p}^{2}}\left\{1-\left[\frac{\alpha_{0}^{2} \pi}{4 A} \ln \left(\frac{A}{4 \ell_{p}^{2}}\right)+\frac{A \beta_{0}^{2}}{2 \pi}\right] \ell_{p}^{2}+\mathcal{O}\left(\ell_{p}^{4}\right)\right\} \\
& =\frac{A c^{3}}{G \hbar}\left\{1-\left[\frac{\alpha_{0}^{2} \pi}{4 A} \ln \left(\frac{A}{4 \ell_{p}^{2}}\right)+\frac{A \beta_{0}^{2}}{2 \pi}\right] \ell_{p}^{2}\right. \\
& \left.+\mathcal{O}\left(\ell_{p}^{4}\right)\right\},
\end{aligned}
$$

where $\ell_{p}^{2}=G \hbar / c^{3}$. By setting the total energy of the black hole (or holographic system) as $E$ and noting that the energy is divided evenly over the bits $N$, it is easy to obtain the notion that each bit carries an energy equal to $k_{B} T / 2$. According to the equipartition rule, the total energy can be expressed as

$$
E=\frac{k_{B} N T}{2} .
$$

Using the relation $E=M c^{2}$ and then putting (12) and (13) into the abovementioned equation, one gets

$$
F=\frac{4 \pi c^{3} M m}{\hbar N} .
$$

Next, substituting $N$ from (15) into (17), the GUP corrected Newton's law of gravitation becomes

$$
\begin{aligned}
F & =\frac{G M m}{R^{2}}\left\{1+\left[\frac{\alpha_{0}^{2}}{16 R} \ln \left(\frac{\pi R}{\ell_{p}^{2}}\right)+2 \beta_{0}^{2} R\right] \ell_{p}^{2}\right. \\
& \left.+\mathcal{O}\left(\ell_{p}^{4}\right)\right\} .
\end{aligned}
$$

In the abovementioned equation, one has $A=4 \pi R^{2}$. It is well known that the Newtonian gravitational force dominates at large scales; however, it becomes weak at small scales (recent experiments show that the Newtonian gravitational force is led down to $0.13 \mathrm{~mm} \sim 0.16 \mathrm{~mm}$ [68]). Meanwhile, it is hard to combine the Newtonian gravitational force with quantum mechanics. In (18), it is clear that the GUP corrected Newton's law is dependent not only on Newton's gravitational constant $G$, the mass of two bodies $M$ and $m$, and the distances $R$ but also on the dimensionless constants $\alpha_{0}$ and $\beta_{0}$ as well as the Planck length $\ell_{p}$. Therefore, due to the effect of GUP, the result shows that the Newtonian gravitational force is valid at scales which is smaller than the order of a millimeter. When $\alpha_{0}=\beta_{0}=0$, (18) reduces to the original Newton's law. Moreover, one can obtain the Newtonian potential from (18):

$$
\begin{aligned}
& V(R)=-\frac{G M m}{R}\{1 \\
& +\left\{\frac{\alpha_{0}^{2}}{32 R}\left[\frac{1}{32}-\ln \left(\frac{\pi R}{\ell_{p}^{2}}\right)\right]-2 R \beta_{0}^{2} \ln R\right\} \ell_{p}^{2} \\
& \left.+\mathcal{O}\left(\ell_{p}^{4}\right)\right\} .
\end{aligned}
$$

It is interesting to compare (19) with the predictions that came from higher order corrections to the Newtonian potential in the Randall-Sundrum II (RS II) [69]; the modification in Newton's gravitational potential on brane is [70]

$$
V(R) \sim \begin{cases}\frac{G M m}{r}\left(1+\frac{4 l_{\mu}}{3 \pi r}-\cdots\right) & \text { for } l_{\mu} \gg r \\ \frac{G M m}{r}\left(1+\frac{2 l_{\mu}}{3 \pi r^{2}}-\cdots\right) & \text { for } l_{\mu} \ll r,\end{cases}
$$

where $r$ and $l_{\mu}$ are the radius and the characteristic length scale of the theory, respectively. Our result agrees with the Newtonian potential in RS II when $l_{\mu} \gg r$. Hence, it suggests that $\left(\alpha_{0}, \beta_{0}\right) \sim l_{\mu}$ can help us to set a new upper bound on the dimensionless constants $\left(\alpha_{0}, \beta_{0}\right)$. Besides, both (20) and the correction terms in (19) become susceptible at a short distance; they indicate that GUP and brane world may predict the similar phenomena.

\section{The Quantum Corrections to Einstein's Field Equation}

A lot of works predict that Einstein's field equation can be derived from entropic force. In this section, we will further investigate the laws of gravity and extend them to the relativistic case, so that we can obtain the modified Einstein's field equation via the GUP corrected entropic force. According to the GUP corrected number of bits, the bit density on the screen can be expressed as

$$
d N=\frac{1}{\ell_{p}^{2}}\left[1-\left(\frac{\alpha_{0}^{2} \pi}{4 A}+\frac{A \beta_{0}^{2}}{\pi}\right) \ell_{p}^{2}+\mathcal{O}\left(\ell_{p}^{4}\right)\right] d A,
$$

where $A$ represents the area of the holographic screen, and we use the natural units $c=k_{B}=1$ in the equation above. 
Here, we assume that the energy associated with the mass $M$ is divided over $N$. Moreover, due to the equipartition law, it is easy to find that each bit carries $T / 2$ mass. Therefore, the total mass is

$$
M=\frac{1}{2} \int_{\mathcal{S}} T d N
$$

where $\mathcal{S}$ is the holographic screen. The local temperature can be expressed as

$$
T=\frac{\hbar e^{\phi} n^{b} \nabla_{b} \varphi}{2 \pi}
$$

where $e^{\phi}$ is the redshift factor as the local temperature is measured by an observer from infinity [71]. Substituting (21) and (23) into (22), one has

$$
\begin{aligned}
M & =\frac{1}{4 \pi G} \\
& \cdot \int_{\mathcal{S}} e^{\phi} \nabla \phi\left[1-\left(\frac{\alpha_{0}^{2} \pi}{4 A}+\frac{A \beta_{0}^{2}}{\pi}\right) \ell_{p}^{2}+\mathcal{O}\left(\ell_{p}^{4}\right)\right] d A .
\end{aligned}
$$

It is necessary to mention that the integral on the right side of the equation above represents the modified Komar mass (the original Komar mass contained inside a volume in a static curved space time is defined as $\left.M_{K}=(1 / 4 \pi G) \int_{\mathcal{S}} e^{\phi} \nabla \phi d A\right)$ $[21,71,72]$; hence, $(24)$ is the modified Gauss law in general relativity. Using the Stokes theorem and the Killing equation $\nabla^{a} \nabla_{a} \xi^{b}=-R_{a}^{b} \xi^{a}$, the Komar mass in terms of the Killing vector $\xi^{a}$ and Ricci tensor $R_{a b}$ can be rewritten as $[21,73]$

$$
M_{K}=\frac{1}{4 \pi G} \int_{\Sigma} R_{a b} n^{a} \xi^{b} d V
$$

Therefore, (24) becomes

$$
\begin{aligned}
M= & \frac{1}{4 \pi G} \int_{\Sigma} R_{a b} n^{a} \xi^{b} d V+\frac{1}{4 \pi G} e^{\phi} \\
& \nabla \phi \int_{\mathcal{S}}\left[-\left(\frac{\alpha_{0}^{2} \pi}{4 A}+\frac{A \beta_{0}^{2}}{\pi}\right) \ell_{p}^{2}+\mathcal{O}\left(\ell_{p}^{4}\right)\right] d A,
\end{aligned}
$$

where $\Sigma$ is the three-dimensional volume bounded by the holographic screen $\delta$ and its normal is $n^{a}$. In [72], the authors showed that $M$ can be expressed in terms of the stress-energy tensor $T_{a b}$ :

$$
M=2 \int_{\Sigma} d V\left(T_{\mu \nu}-\frac{1}{2} T g_{\mu \nu}\right) n^{a} \xi^{b}
$$

As a result, substituting (27) into (26), one yields

$$
\begin{aligned}
& \int_{\Sigma}\left[R_{a b}-8 \pi G\left(T_{a b}-\frac{1}{2} T g_{a b}\right)\right] n^{a} \xi^{b} d V \\
& =\ell_{p}^{2} \int_{\mathcal{S}} e^{\phi} \nabla \phi\left[\frac{\alpha_{0}^{2} \pi}{4 A}+\frac{A \beta_{0}^{2}}{\pi}+\mathcal{O}\left(\ell_{p}^{4}\right)\right] d A .
\end{aligned}
$$

With the help of $F=-m e^{\phi} \nabla \phi$, one can obtain the GUP corrected Einstein's field equation by some manipulations:

$$
\begin{aligned}
R_{a b}= & 8 \pi G\left(T_{a b}-\frac{1}{2} T g_{a b}\right) \\
& \cdot\left[1-\left(\frac{\alpha_{0}^{2} \pi}{4 A}+\frac{A \beta_{0}^{2}}{\pi}\right) \frac{\ell_{p}^{2}}{2 \pi}+\mathcal{O}\left(\ell_{p}^{4}\right)\right],
\end{aligned}
$$

with the area of the holographic screen $A$. Obviously, this field equation is dependent not only on the geometry of the space time and energy-momentum tensor but also on the GUP terms. For large horizon area or $\alpha_{0}=\beta_{0}=0$, the modified Einstein's field equation reduces to the original case.

\section{The Quantum Corrections to the Friedmann Equation}

In $[19,22,51,73-76]$, people analyzed the Friedmann equation by using the entropic force. Hence, we will study the effect of the GUP arising from (1) on the form of the Friedmann equation. In the homogeneous and isotropic spacetime, the Friedmann-Robertson-Walker (FRW) universe is described by the line element:

$$
d s^{2}=h_{\mu \nu} d x^{\mu} d x^{\nu}+\tilde{r}^{2} d \Omega^{2}
$$

where $\tilde{r}=r a(t), x^{\mu}=(t, r), d \Omega^{2}=d \theta^{2}+\sin ^{2} \theta d \varphi^{2}$ is the metric of two-dimensional unit sphere, $h_{\mu \nu}=\operatorname{diag}\left[-1, a^{2} /(1-\right.$ $\left.k r^{2}\right)$ ] is the two-dimensional metric with $\mu=\nu=0,1$, and $k$ is the spatial curvature constant, respectively. Using the relation $h^{\mu \nu} \partial_{\mu} \tilde{r} \partial_{\gamma} \tilde{r}=0$, the dynamical apparent horizon of the FRW universe can be expressed as

$$
\widetilde{r}=a r=\frac{1}{\sqrt{H^{2}+k / a^{2}}},
$$

where $H=\dot{a} / a$ is the Hubble parameter. Now, supposing that the matter source in the FRW universe is a perfect fluid, stress-energy tensor is

$$
T_{\mu \nu}=(\rho+p) u_{\mu} u_{\nu}+p g_{\mu \nu}
$$

where $u_{\mu}$ is the four velocities of the fluid. The conservation law of energy-momentum leads to the following continuity equation:

$$
\dot{\rho}+3 H(\rho+p)=0 .
$$

In order to investigate the GUP corrected Friedmann equation, one should consider a compact spatial region $V=$ $(4 / 3) \pi \widetilde{r}^{3}$ with a compact boundary $\Sigma=4 \pi \widetilde{r}^{2}$. By combining (18) with Newton's second law, one has

$$
F=m\left(\frac{\partial^{2} \widetilde{r}}{\partial t^{2}}\right)=m \ddot{a} r=-G \frac{M m}{\widetilde{r}^{2}} \chi\left(\ell_{p}, \alpha_{0}, \beta_{0}\right),
$$

where $\chi\left(\ell_{p}, \alpha_{0}, \beta_{0}\right)=1+\left[2 \beta_{0}^{2} \widetilde{r}^{2}+\alpha_{0}^{2} \ln \left(\pi \widetilde{r}^{2} / \ell_{p}^{2}\right) / 16 \widetilde{r}^{2}\right] \ell_{p}^{2}+$ $\mathcal{O}\left(\ell_{p}^{4}\right)$ and $m$ represents the test particle near the holographic 
screen; the higher order terms $\mathcal{O}\left(\ell_{p}^{4}\right)$ can be ignored since $\ell_{p}$ is a very small value. The total physical mass $M$ inside the volume $\mathscr{V}$ can be defined as

$$
M=\int_{\mathscr{V}} d V\left(T_{\mu \nu} u^{\mu} u^{\nu}\right)=\frac{4}{3} \pi \tilde{r}^{3} \rho,
$$

where $\rho=M / V$ is the energy density of the matter in the spatial region $V$. Putting (34) into (33), one has the acceleration equation:

$$
\frac{\ddot{a}}{a}=-\frac{4}{3} \pi G \rho \chi\left(\ell_{p}, \alpha_{0}, \beta_{0}\right) .
$$

For deriving the Friedmann equation, it is necessary to use the active gravitational mass (or Tolman-Komar mass) $\mathscr{M}$ instead of the total mass $M$ because the acceleration in a dynamical background is produced by the active gravitational mass. According to [22], one can express the active gravitational mass in terms of energy-momentum tensor $T_{\mu \nu}$ :

$$
\mathscr{M}=2 \int_{\mathscr{V}} d V\left(T_{\mu \nu}-\frac{1}{2} T g_{\mu \nu}\right) u^{\mu} u^{\nu}=\frac{4}{3} \pi \tilde{r}^{3}(\rho+3 p)
$$

Replacing the total mass $M$ by the active gravitational mass $\mathscr{M}$, (35) can be rewritten as

$$
\frac{\ddot{a}}{a}=-\frac{4}{3} \pi G(\rho+3 p) \chi\left(\ell_{p}, \alpha_{0}, \beta_{0}\right) .
$$

The equation above is the GUP corrected acceleration equation for the dynamical evolution of the FRW universe. Using continuity equation (32) and multiplying both sides of (37) with $\dot{a} a$ and then integrating it, the result is [51]

$$
\frac{d}{d t}\left(\dot{a}^{2}\right)=\frac{8 \pi G}{3}\left[\frac{d}{d t}\left(\rho a^{2}\right)\right] \chi\left(\ell_{p}, \alpha_{0}, \beta_{0}\right) .
$$

Integrating both sides for each term of (38), one has

$$
\begin{aligned}
& \dot{a}^{2}+k=\frac{8 \pi G}{3} \rho a^{2}\left\{1+\frac{1}{\rho a^{2}}\right. \\
& \cdot \int\left[2(r a)^{2} \beta_{0}^{2}+\frac{\alpha_{0}^{2} \pi}{16 \pi(r a)^{2}} \ln \left(\frac{\pi(r a)^{2}}{\ell_{p}^{2}}\right)\right] \\
& \left.\cdot \ell_{p}^{2} d\left(\rho a^{2}\right)\right\} ;
\end{aligned}
$$

the above equation can be rewritten as

$$
\begin{aligned}
& H^{2}+\frac{k}{a^{2}}=\frac{8 \pi G}{3} \rho\left\{1+\frac{1}{\rho a^{2}}\right. \\
& \cdot \int\left[2(r a)^{2} \beta_{0}^{2}+\frac{\alpha_{0}^{2} \pi}{16 \pi(r a)^{2}} \ln \left(\frac{\pi(r a)^{2}}{\ell_{p}^{2}}\right)\right] \\
& \left.\cdot \ell_{p}^{2} d\left(\rho a^{2}\right)\right\} .
\end{aligned}
$$

It should be noted that $k$ is the spatial curvature which takes the values $-1,0,1$, and the values correspond to a close, flat, and open FRW universe, respectively. For calculating the correction term of (40), we assume an equation of state parameter is $\omega=p / \rho$, where $\omega$ is a constant independent of time (or redshift), so, integrating the continuity equation (33), it yields

$$
\rho=\rho_{0} a^{-3(1+\omega)}
$$

where $\rho_{0}$ is an integration constant. Substituting (41) into (40) and integrating, the result is given by

$$
\begin{gathered}
H^{2}+\frac{k}{a^{2}}=\frac{8 \pi G}{3} \rho\left\{1+(1+3 \omega)\left[\frac{\alpha_{0}^{2}}{72 \widetilde{r}^{2}(1+\omega)^{2}}\right.\right. \\
\left.\left.+\frac{2 \beta_{0}^{2} \widetilde{r}^{2}}{3 \omega-1}+\frac{\alpha_{0}^{2}}{24 \widetilde{r}^{2}(1+\omega)} \ln \left(\frac{\tilde{r} \sqrt{\pi}}{\ell_{p}}\right)\right] \ell_{p}^{2}\right\} .
\end{gathered}
$$

With the help of (31), the equation above can be further rewritten as

$$
\begin{aligned}
& \left(H^{2}+\frac{k}{a^{2}}\right)\{1-(1+3 \omega) \\
& \cdot \ell_{p}^{2}\left[\frac{\alpha_{0}^{2}}{72(1+\omega)^{2}}\left(H^{2}+\frac{k}{a^{2}}\right)\right. \\
& +\frac{2 \beta_{0}^{2}}{3 \omega-1}\left(H^{2}+\frac{k}{a^{2}}\right)^{-1} \\
& \left.\left.+\frac{\alpha_{0}^{2}}{24(1+\omega)}\left(H^{2}+\frac{k}{a^{2}}\right) \ln \left[\left(H^{2}+\frac{k}{a^{2}}\right) \frac{\ell_{p}^{2}}{\pi}\right]\right]\right\} \\
& =\frac{8 \pi G}{3} \rho .
\end{aligned}
$$

The equation above is the GUP corrected Friedmann equation of the FRW universe, which derived from the entropic force. It should be noted that (43) is not only determined by the Hubble parameter $H$, the expansion scale factor of universe $a$, the spatial curvature $k$, and the constant $\omega$ but also affected by the dimensionless constants $\alpha_{0}$ and $\beta_{0}$ and the Planck length $\ell_{p}$. For the present universe, (43) is nothing but a usual Friedmann equation since the apparent horizon radius is very large. However, the correction terms make sense when the apparent horizon radius is at a short scale. Hence, one can use the GUP corrected Friedmann to investigate the early stage of the universe. Moreover, in $[51,52]$, the authors concluded that the impact of quantum corrections at the early stage of the universe can affect the inflation, so that people may detect those consequences by astronomical observation.

\section{Conclusions}

In this paper, we studied the quantum corrections to the entropic force via a new kind of GUP that admits a minimal 
length, a minimal momentum, and a maximal momentum. Firstly, we derived the modified entropy-area law of a black hole. Then, using the the modification of entropy, the GUP corrected number of bits $N$ was obtained. Subsequently, based on the GUP corrected number of bits and Verlinde's conjecture about the entropic force, the GUP corrected Newton's law of gravitation and Einstein's field equation as well as the Friedmann equation have been investigated. The results showed that the GUP corrected Newton's law of gravitation, the GUP corrected Einstein's field equation, and the GUP corrected Friedmann equation are dependent on the quantum correction terms $\alpha_{0}$ and $\beta_{0}$ and the Planck length $\ell_{p}$. These results agree with the original cases at a larger scale. However, when the length approaches the order of Planck scale, the corrected results depart from the original cases since the GUP effect becomes susceptible at a short scale. Besides, it is found that the GUP corrected Newton's law of gravitation is working at the sub- $\mu \mathrm{m}$ range, and it can predict the similar phenomenon as the Randall-Sundrum II model; this can help us to set a new upper bound on $\left(\alpha_{0}, \beta_{0}\right)$. Moreover, we found that the GUP corrected Friedmann equation can help people to study the properties of the early universe, and the impact of GUP may be detected by the astronomical observation.

\section{Competing Interests}

The authors declare that they have no competing interests.

\section{Acknowledgments}

The authors would like to acknowledge useful discussions with Karissa Liu and Lorraine Boyd. This work is supported by the Natural Science Foundation of China (Grant no. 11573022).

\section{References}

[1] F. R. Tangherlini, "Schwarzschild field in $n$ dimensions and the dimensionality of space problem," ILNuovo Cimento, vol. 27, pp. 636-651, 1963.

[2] J. D. Bekenstein, "Black holes and entropy," Physical Review. D. Particles and Fields. Third Series, vol. 7, pp. 2333-2346, 1973.

[3] J. D. Bekenstein, "Generalized second law of thermodynamics in black-hole physics," Physical Review D, vol. 9, no. 12, pp. 32923300, 1974.

[4] S. W. Hawking, "Black hole explosions?” Nature, vol. 248, no. 5443, pp. 30-31, 1974.

[5] S. W. Hawking, "Particle creation by black holes," Communications in Mathematical Physics, vol. 43, no. 3, pp. 199-220, 1975.

[6] D. Y. Chen, H. T. Yang, and X. T. Zu, "Fermion tunneling from anti-de Sitter spaces," The European Physical Journal C, vol. 56, no. 1, pp. 119-124, 2008.

[7] W. G. Unruh, "Notes on black-hole evaporation," Physical Review D, vol. 14, no. 4, pp. 870-892, 1976.

[8] M. Li and T. Yoneya, "Short-distance space-time structure and black holes in string theory: a short review of the present status," Chaos, Solitons \& Fractals, vol. 10, no. 2-3, pp. 423-443, 1999.
[9] J. M. Bardeen, B. Carter, and S. W. Hawking, "The four laws of black hole mechanics," Communications in Mathematical Physics, vol. 31, pp. 161-170, 1973.

[10] D. Y. Chen, H. T. Yang, and X. T. Zu, "Area spectra of near extremal black holes," The European Physical Journal C, vol. 69, no. 1, pp. 289-292, 2010.

[11] G.-M. Deng, "Hawking radiation of charged rotating AdS black holes in conformal gravity for charged massive particles, complex scalar and Dirac particles," General Relativity and Gravitation, vol. 46, no. 6, Article ID 1757, 2014.

[12] T. Jacobson, "Thermodynamics of spacetime: the Einstein equation of state," Physical Review Letters, vol. 75, no. 7, pp. 12601263, 1995.

[13] T. Padmanabhan, "Classical and quantum thermodynamics of horizons in spherically symmetric spacetimes," Classical and Quantum Gravity, vol. 19, no. 21, pp. 5387-5408, 2002.

[14] A. Paranjape, S. Sarkar, and T. Padmanabhan, "Thermodynamic route to field equations in Lanczos-Lovelock gravity," Physical Review. D. Third Series, vol. 74, no. 10, Article ID 104015, 2006.

[15] D. Kothawala, S. Sarkar, and T. Padmanabhan, "Einstein's equations as a thermodynamic identity: the cases of stationary axisymmetric horizons and evolving spherically symmetric horizons," Physics Letters B, vol. 652, no. 5-6, pp. 338-342, 2007.

[16] R.-G. Cai and L.-M. Cao, "Thermodynamics of apparent horizon in brane world scenario," Nuclear Physics B, vol. 785, no. 1-2, pp. 135-148, 2007.

[17] R.-G. Cai and N. Ohta, "Horizon thermodynamics and gravitational field equations in Hŏrava-Lifshitzgravity," Physical Review D, vol. 81, no. 8, Article ID 084061, 8 pages, 2010.

[18] T. Padmanabhan, "Thermodynamical aspects of gravity: new insights," Reports on Progress in Physics, vol. 73, no. 4, Article ID 046901, 2010.

[19] S. H. Hendi and A. Sheykhi, "Entropic corrections to Einstein equations," Physical Review D, vol. 83, no. 8, Article ID 084012, 2011.

[20] A. D. Sakharov, "Vacuum quantum fluctuations in curved space and the theory of gravitation," General Relativity and Gravitation, vol. 32, no. 2, pp. 365-367, 2000.

[21] E. Verlinde, "On the origin of gravity and the laws of Newton," Journal of High Energy Physics, vol. 4, article 29, 27 pages, 2011.

[22] R.-G. Cai, L.-M. Cao, and N. Ohta, "Friedmann equations from entropic force," Physical Review D-Particles, Fields, Gravitation and Cosmology, vol. 81, no. 6, Article ID 061501, 2010.

[23] I. Sakalli, "Dilatonic entropic force," International Journal of Theoretical Physics, vol. 50, no. 8, pp. 2426-2437, 2011.

[24] Y. S. Myung, "Does entropic force always imply the Newtonian force law?” The European Physical Journal C, vol. 71, no. 2, article 1549, 2011.

[25] R. G. L. Aragõo and C. A. S. Silva, “Entropic corrected Newton's law of gravitation and the loop quantum black hole gravitational atom," General Relativity and Gravitation, vol. 48, p. 83, 2016.

[26] Y. S. Myung, Y.-W. Kim, and Y.-J. Park, "Black hole thermodynamics with generalized uncertainty principle," Physics Letters $B$, vol. 645, no. 5-6, pp. 393-397, 2007.

[27] D. Y. Chen, H. W. Wu, and H. T. Yang, "Fermion's tunnelling with effects of quantum gravity," Advances in High Energy Physics, vol. 2013, Article ID 432412, 6 pages, 2013.

[28] D. Chen and Z. Li, "Remarks on remnants by fermions' tunnelling from black strings," Advances in High Energy Physics, vol. 2014, Article ID 620157, 9 pages, 2014. 
[29] Z. W. Feng, L. Zhang, and X. T. Zu, "The remnants in ReissnerNordström-de Sitter quintessence black hole," Modern Physics Letters A, vol. 29, no. 26, Article ID 1450123, 2014.

[30] Z. W. Feng, H. L. Li, X. T. Zu, and S. Z. Yang, "Quantum corrections to the thermodynamics of Schwarzschild-Tangherlini black hole and the generalized uncertainty principle," The European Physical Journal C, vol. 76, article 212, 2016.

[31] A. Ashour, M. Faizal, A. F. Ali, and F. Hammad, "Branes in Gravity's rainbow," The European Physical Journal C, vol. 76, article 264, 2016.

[32] F. Scardigli, "Generalized uncertainty principle in quantum gravity from micro-black hole Gedanken experiment," Physics Letters B, vol. 452, no. 1-2, pp. 39-44, 1999.

[33] J. Magueijo and L. Smolin, "Generalized Lorentz invariance with an invariant energy scale," Physical Review D, vol. 67, no. 4, Article ID 044017, 12 pages, 2003.

[34] J. Magueijo and L. Smolin, "Gravity's rainbow," Classical and Quantum Gravity, vol. 21, no. 7, pp. 1725-1736, 2004.

[35] S. Das and E. C. Vagenas, "Universality of quantum gravity corrections," Physical Review Letters, vol. 101, no. 22, Article ID 221301, 2008.

[36] A. Ashoorioon, A. Kempf, and R. B. Mann, "Minimum length cutoff in inflation and uniqueness of the action," Physical Review D-Particles, Fields, Gravitation and Cosmology, vol. 71, Article ID 023503, 2005.

[37] A. Ashoorioon and R. B. Mann, "On the tensor/scalar ratio in inflation with UV cutoff," Nuclear Physics B, vol. 716, no. 1-2, pp. 261-279, 2005.

[38] A. Ashoorioon, J. L. Hovdebo, and R. B. Mann, "Running of the spectral index and violation of the consistency relation between tensor and scalar spectra from trans-Planckian physics," Nuclear Physics B, vol. 727, no. 1-2, pp. 63-76, 2005.

[39] S. Das and R. B. Mann, "Planck scale effects on some low energy quantum phenomena," Physics Letters B, vol. 704, no. 5, pp. 596599, 2011.

[40] M. Sprenger, P. Nicolini, and M. Bleicher, "Neutrino oscillations as a novel probe for a minimal length," Classical and Quantum Gravity, vol. 28, no. 23, Article ID 235019, 2011.

[41] A. F. Ali, S. Das, and E. C. Vagenas, "Proposal for testing quantum gravity in the lab," Physical Review D-Particles, Fields, Gravitation and Cosmology, vol. 84, Article ID 044013, 2011.

[42] T. Zhu, J.-R. Ren, and M.-F. Li, "Influence of generalized and extended uncertainty principle on thermodynamics of FRW universe," Physics Letters B, vol. 674, no. 3, pp. 204-209, 2009.

[43] W. Chemissany, S. Das, A. F. Ali, and E. C. Vagenas, "Effect of the generalized uncertainty principle on post-inflation preheating," Journal of Cosmology and Astroparticle Physics, vol. 2011, no. 12, p. $17,2011$.

[44] M. Asghari, P. Pedram, and K. Nozari, "Harmonic oscillator with minimal length, minimal momentum, and maximal momentum uncertainties in SUSYQM framework," Physics Letters B, vol. 725, no. 4-5, pp. 451-455, 2013.

[45] F. Scardigli and R. Casadio, "Gravitational tests of the generalized uncertainty principle," The European Physical Journal C, vol. 75, article 425, 2015.

[46] F. Scardigli, "Generalized uncertainty principle in quantum gravity from micro-black hole gedanken experiment," Physics Letters, Section B: Nuclear, Elementary Particle and High-Energy Physics, vol. 452, no. 1-2, pp. 39-44, 1999.

[47] F. Scardigli, "Glimpses on the micro black hole Planck phase," https://arxiv.org/abs/0809.1832.
[48] P. Jizba, H. Kleinert, and F. Scardigli, "Uncertainty relation on a world crystal and its applications to micro black holes," Physical Review D, vol. 81, no. 8, Article ID 084030, 13 pages, 2010.

[49] R. J. Adler, P. Chen, and D. I. Santiago, "The generalized uncertainty principle and black hole remnants," General Relativity and Gravitation, vol. 33, no. 12, pp. 2101-2108, 2001.

[50] S. Ghosh, "Planck scale effect in the entropic force Law," http:// arxiv.org/abs/1003.0285.

[51] A. Awad and A. F. Ali, "Planck-scale corrections to Friedmann equation," Central European Journal of Physics, vol. 12, no. 4, pp. 245-255, 2014.

[52] P. Jizba, H. Kleinert, and F. Scardigli, "Inflationary cosmology from quantum conformal gravity," European Physical Journal C, vol. 75, no. 6, article 245, 2015.

[53] A. Awad and A. F. Ali, "Minimal length, Friedmann equations and maximum density," Journal of High Energy Physics, vol. 2014, article 93, 2014.

[54] K. Nozari, P. Pedram, and M. Molkara, "Minimal length, maximal momentum and the entropic force law," International Journal of Theoretical Physics, vol. 51, no. 4, pp. 1268-1275, 2012.

[55] M. M. Amaral, R. Aschheim, and K. Irwin, "Quantum walk on spin network," https://arxiv.org/abs/1602.07653.

[56] D. E. Kharzeev, "Deconfinement as an entropic self-destruction: a solution for the quarkonium suppression puzzle?" Physical Review D, vol. 90, no. 7, Article ID 074007, 7 pages, 2014.

[57] H. Satz, "Quarkonium binding and entropic force," The European Physical Journal C, vol. 75, article 193, 2015.

[58] P. Pedram, K. Nozari, and S. H. Taheri, “The effects of minimal length and maximal momentum on the transition rate of ultra cold neutrons in gravitational field," Journal of High Energy Physics, vol. 2011, no. 3, article 093, 2011.

[59] H. Soltani, A. Damavandi Kamali, and K. Nozari, "Microblack holes thermodynamics in the presence of quantum gravity effects," Advances in High Energy Physics, vol. 2014, Article ID 247208, 12 pages, 2014.

[60] K. Nozari and S. Saghafi, "Natural cutoffs and quantum tunneling from black hole horizon," Journal of High Energy Physics, vol. 2012, article 5, 2012.

[61] F. Scardigli, "Some heuristic semi-classical derivations of the Planck length, the Hawking effect and the Unruh effect," Il Nuovo Cimento B, vol. 110, no. 9, pp. 1029-1034, 1995.

[62] A. J. Medved and E. C. Vagenas, "When conceptual worlds collide: the generalized uncertainty principle and the BekensteinHawking entropy," Physical Review D, vol. 70, no. 12, Article ID 124021, 5 pages, 2004.

[63] C. Adami, "The physics of information," Philosophy of Information, vol. 42, pp. 609-683, 2004.

[64] M.-I. Park, “The generalized uncertainty principle in (A)dS space and the modification of Hawking temperature from the minimal length," Physics Letters B, vol. 659, no. 3, pp. 698-702, 2008.

[65] R. Banerjee and B. R. Majhi, "Quantum tunneling and back reaction," Physics Letters B, vol. 662, no. 1, pp. 62-65, 2008.

[66] P. Bargueño and E. C. Vagenas, "Semiclassical corrections to black hole entropy and the generalized uncertainty principle," Physics Letters B, vol. 742, pp. 15-18, 2015.

[67] G. t'Hooft, “Dimensional reduction in quantum gravity," https:// arxiv.org/abs/gr-qc/9310026.

[68] S.-Q. Yang, B.-F. Zhan, Q.-L. Wang et al., "Test of the gravitational inverse square law at millimeter ranges," Physical Review Letters, vol. 108, no. 8, Article ID 081101, 2012. 
[69] L. Randall and R. Sundrum, "An alternative to compactification," Physical Review Letters, vol. 83, no. 23, pp. 4690-4693, 1999.

[70] P. Callin and F. Ravndal, "Higher order corrections to the Newtonian potential in the Randall-Sundrum model," Physical Review D, vol. 70, no. 10, Article ID 104009, 2004.

[71] B. Majumder, "The effects of minimal length in entropic force approach," Advances in High Energy Physics, vol. 2013, Article ID 296836, 8 pages, 2013.

[72] A. S. Sefiedgarr, "How can rainbow gravity affect on gravitational force?" http://arxiv.org/abs/1512.08372.

[73] R. G. Cai and S. P. Kim, "First law of thermodynamics and Friedmann equations of Friedmann Robertson Walker universe," Journal of High Energy Physics, vol. 2, article 50, 2005.

[74] A. Sheykhi, "Entropic corrections to Friedmann equations," Physical Review D, vol. 81, no. 10, Article ID 104011, 2010.

[75] Y. F. Cai, J. Liu, and H. Li, "Entropic cosmology: a unified model of inflation and late-time acceleration," Physics Letters B, vol. 697, pp. 213-219, 2010.

[76] Y.-F. Cai and E. N. Saridakis, "Inflation in entropic cosmology: primordial perturbations and non-Gaussianities," Physics Letters B, vol. 697, no. 4, pp. 280-287, 2011. 

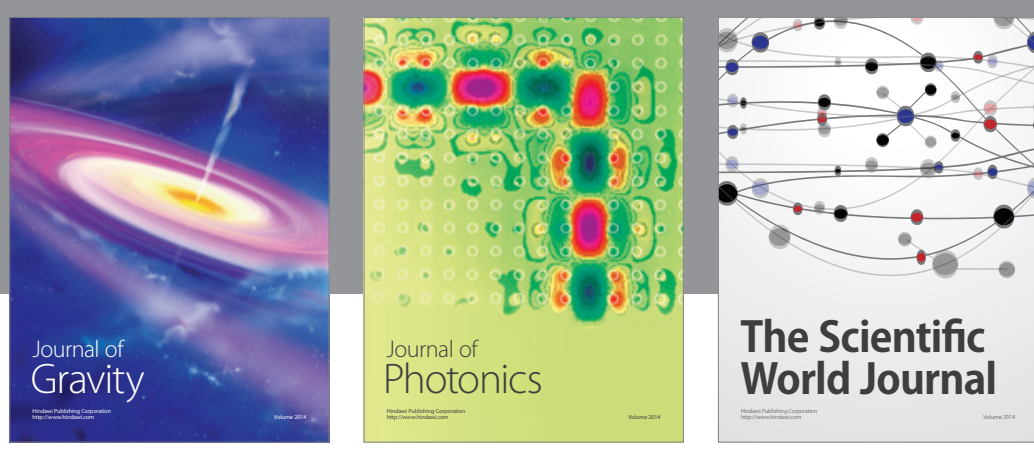

The Scientific World Journal
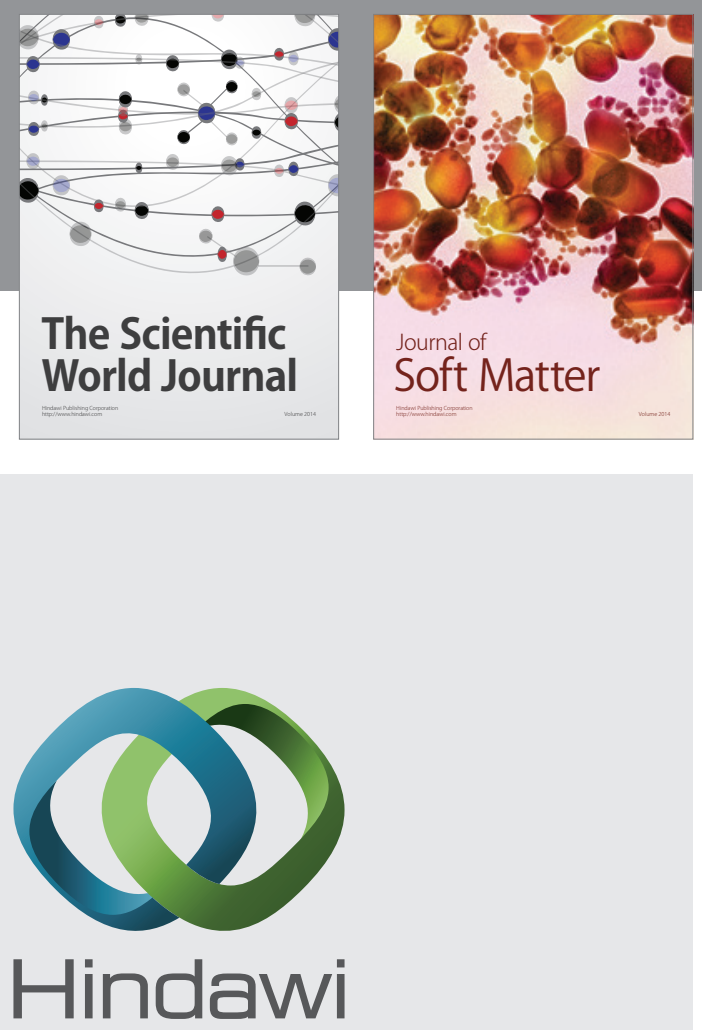

Submit your manuscripts at

http://www.hindawi.com

nternational Journal of

Statistical Mechanics
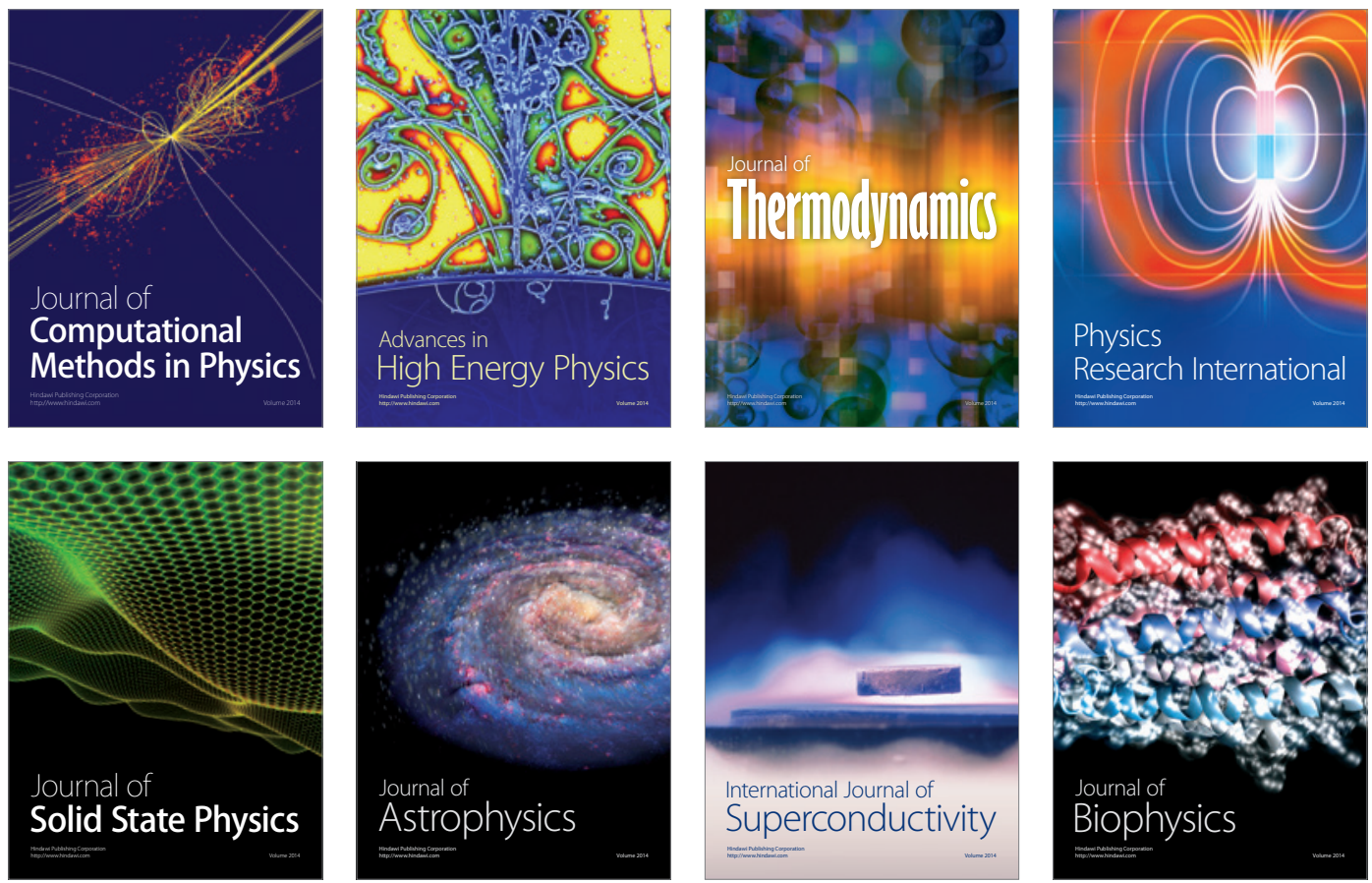
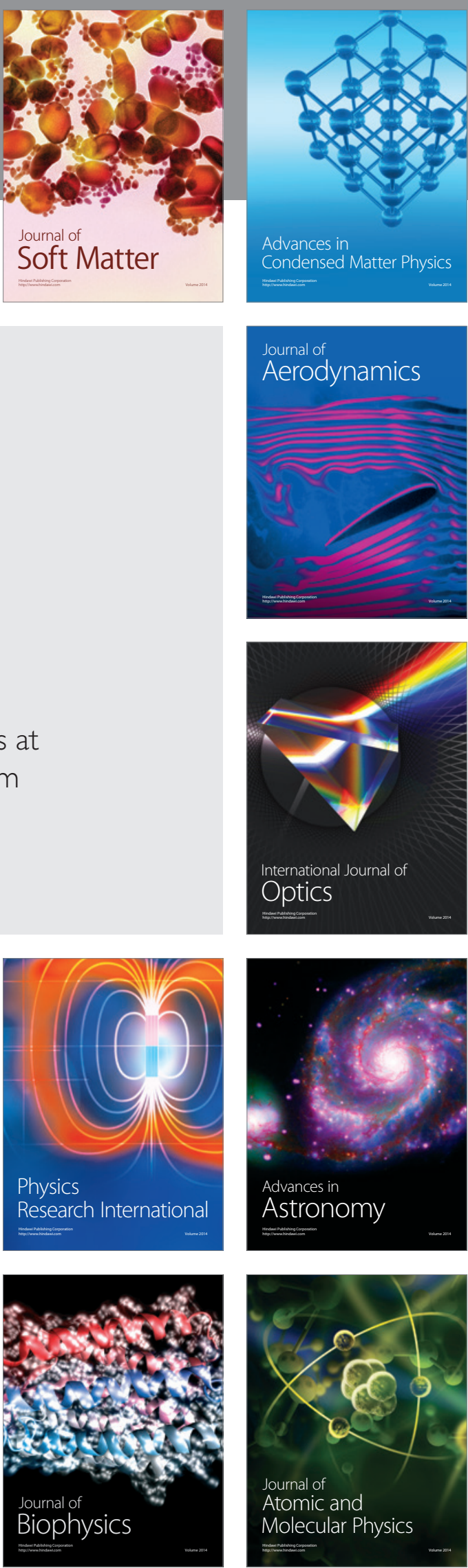